\title{
New proofs of Schur-concavity for a class of symmetric functions
}

\author{
Huan-Nan Shi*, Jian Zhang and Chun Gu
}

* Correspondence:

shihuannan@yahoo.com.cn

Department of Electronic

Information, Teacher's College,

Beijing Union University, Beijing

100011, P.R. China

\section{Abstract}

By properties of the Schur-convex function, Schur-concavity for a class of symmetric functions is simply proved uniform.

2000 Mathematics Subject Classification: Primary 26D15; 05E05; 26B25.

Keywords: majorization, Schur-concavity, inequality, symmetric functions, concave functions

\section{Introduction}

Throughout the article, $\mathbb{R}$ denotes the set of real numbers, $\boldsymbol{x}=\left(x_{1}, x_{2}, \ldots, x_{n}\right)$ denotes $n$-tuple (n-dimensional real vectors), the set of vectors can be written as

$$
\begin{aligned}
& \mathbb{R}^{n}=\left\{x=\left(x_{1}, \ldots, x_{n}\right): x_{i} \in \mathbb{R}, i=1, \ldots, n\right\}, \\
& \mathbb{R}_{+}^{n}=\left\{x=\left(x_{1}, \ldots, x_{n}\right): x_{i}>0, i=1, \ldots, n\right\} .
\end{aligned}
$$

In particular, the notations $\mathbb{R}$ and $\mathbb{R}_{+}$denote $\mathbb{R}^{1}$ and $\mathbb{R}_{+}^{1}$ respectively.

For convenience, we introduce some definitions as follows.

Definition 1. [1,2] Let $\boldsymbol{x}=\left(x_{1}, \ldots, x_{n}\right)$ and $\boldsymbol{y}=\left(y_{1}, \ldots, y_{n}\right)\left\llcorner\mathbb{R}^{n}\right.$.

(i) $\boldsymbol{x} \geq \boldsymbol{y}$ means $x_{i} \geq y_{i}$ for all $i=1,2, \ldots, n$.

(ii) Let $\Omega \subset \mathbb{R}^{n}, \phi: \Omega \rightarrow \mathbb{R}$ is said to be increasing if $\boldsymbol{x} \geq \boldsymbol{y}$ implies $\phi(\boldsymbol{x}) \geq \phi(\boldsymbol{y})$. $\phi$ is said to be decreasing if and only if $-\phi$ is increasing.

Definition 2. [1,2] Let $\boldsymbol{x}=\left(x_{1}, \ldots, x_{n}\right)$ and $\boldsymbol{y}=\left(y_{1}, \ldots, y_{n}\right) \in \mathbb{R}^{n}$.

(i) $\boldsymbol{x}$ is said to be majorized by $\boldsymbol{y}$ (in symbols $\boldsymbol{x} \prec \boldsymbol{y}$ ) if $\sum_{i=1}^{k} x_{[i]} \leq \sum_{i=1}^{k} \gamma_{[i]}$ for $k=$ $1,2, \ldots, n-1$ and $\sum_{i=1}^{n} x_{i}=\sum_{i=1}^{n} y_{i}$, where $x_{[1]} \geq \cdots \geq x_{[n]}$ and $y_{[1]} \geq \cdots \geq y_{[n]}$ are rearrangements of $\boldsymbol{x}$ and $\boldsymbol{y}$ in a descending order.

(ii) Let $\Omega \subset \mathbb{R}^{n}, \phi: \Omega \rightarrow \mathbb{R}$ is said to be a Schur-convex function on $\Omega$ if $x<\boldsymbol{y}$ on $\Omega$ implies $\phi(\boldsymbol{x}) \leq \phi(\boldsymbol{y})$. $\phi$ is said to be a Schur-concave function on $\Omega$ if and only if $-\phi$ is Schur-convex function on $\Omega$.

Definition 3. [1,2] Let $\boldsymbol{x}=\left(x_{1}, \ldots, x_{n}\right)$ and $\boldsymbol{y}=\left(y_{1}, \ldots, y_{n}\right) \in \mathbb{R}^{n}$. 
(i) $\Omega \subseteq \mathbb{R}^{n}$ is said to be a convex set if $\boldsymbol{x}, \boldsymbol{y} \in \Omega, 0 \leq \alpha \leq 1$ implies $\alpha \boldsymbol{x}+(1-\alpha) \boldsymbol{y}=$ $\left(\alpha x_{1}+(1-\alpha) y_{1}, \ldots, \alpha x_{n}+(1-\alpha) y_{n}\right) \in \Omega$.

(ii) Let $\Omega \subset \mathbb{R}^{n}$ be convex set. A function $\phi: \Omega \rightarrow \mathbb{R}$ is said to be a convex function on $\Omega$ if

$$
\varphi(\alpha x+(1-\alpha) y) \leq \alpha \varphi(x)+(1-\alpha) \varphi(y)
$$

for all $x, y \in \Omega$, and all $\alpha \in[0,1]$. $\phi$ is said to be a concave function on $\Omega$ if and only if $-\phi$ is convex function on $\Omega$.

Recall that the following so-called Schur's condition is very useful for determining whether or not a given function is Schur-convex or Schur-concave.

Theorem A. [[1], p. 5] Let $\Omega \subset \mathbb{R}^{n}$ is symmetric and has a nonempty interior convex set. $\Omega^{0}$ is the interior of $\Omega . \phi: \Omega \rightarrow \mathbb{R}$ is continuous on $\Omega$ and differentiable in $\Omega^{0}$. Then $\phi$ is the Schur-convex (Schur-concave) function, if and only if $\phi$ is symmetric on $\Omega$ and

$$
\left(x_{1}-x_{2}\right)\left(\frac{\partial \varphi}{\partial x_{1}}-\frac{\partial \varphi}{\partial x_{2}}\right) \geq 0(\leq 0)
$$

holds for any $x \in \Omega^{0}$.

In recent years, by using Theorem A, many researchers have studied the Schur-convexity of some of symmetric functions.

Chu et al. [3] defined the following symmetric functions

$$
F_{n}(x, k)=\prod_{1 \leq i_{1}<\ldots<i_{k} \leq n} \frac{\sum_{j=1}^{k} x_{i_{j}}}{\sum_{j=1}^{k}\left(1+x_{i_{j}}\right)}, k=1, \ldots, n,
$$

and established the following results by using Theorem A.

Theorem B. For $k=1, \ldots, n, F_{n}(\boldsymbol{x}, k)$ is an Schur-concave function on $\mathbb{R}_{+}^{n}$.

Jiang [4] are discussed the following symmetric functions

$$
H_{k}^{*}(x)=\prod_{1 \leq i_{1}<\ldots<i_{k} \leq n} \sum_{j=1}^{k} x_{i_{j}}^{1 / k}, k=1, \ldots, n,
$$

and established the following results by using Theorem A.

Theorem C. For $k=1, \ldots, n, H_{k}^{*}(x)$ is an Schur-concave function on $\mathbb{R}_{+}^{n}$.

$\mathrm{Xia}$ and Chu [5] investigated the following symmetric functions

$$
\phi_{n}(x, k)=\prod_{1 \leq i_{1}<\ldots<i_{k} \leq n} \sum_{j=1}^{k} \frac{x_{i_{j}}}{1+x_{i_{j}}}, k=1, \ldots, n,
$$

and established the following results by using Theorem A.

Theorem D. For $k=1, \ldots, n, F_{n}(x, k)$ is an Schur-concave function on $\mathbb{R}_{+}^{n}$.

In this note, by properties of the Schur-convex function, we simply prove Theorems $\mathrm{B}, \mathrm{C}$ and $\mathrm{D}$ uniform.

\section{New proofs three theorems}

To prove the above three theorems, we need the following lemmas.

Lemma 1. [[1], p. 67], [2]If $\phi$ is symmetric and convex (concave) on symmetric convex set $\Omega$, then $\phi$ is Schur-convex (Schur-concave) on $\Omega$. 
Lemma 2. [[1], p. 73],[2]Let $\Omega \subset \mathbb{R}^{n}, \phi: \Omega \rightarrow \mathbb{R}_{+}$. Then $\ln \phi$ is Schur-convex (Schurconcave) if and only if $\phi$ is Schur-convex (Schur-concave).

Lemma 3. [[1], p. 446], [2]Let $\Omega \subset \mathbb{R}^{n}$ be open convex set, $\phi: \Omega \rightarrow \mathbb{R}$. For $\boldsymbol{x}, \boldsymbol{y} \in \Omega$, defined one variable function $g(t)=\phi(t x+(1-t) y)$ on interval $(0,1)$. Then $\phi$ is convex (concave) on $\Omega$ if and only if $g$ is convex (concave) on $(0,1)$ for all $\boldsymbol{x}, \boldsymbol{y}\lfloor\Omega$.

Lemma 4. Let $\boldsymbol{x}=\left(x_{1}, \ldots, x_{m}\right)$ and $\boldsymbol{y}=\left(y_{1}, \ldots, y_{m}\right) \in \mathbb{R}^{m}$. Then the following functions are concave on $(0,1)$.

(i) $f(t)=\ln \sum_{j=1}^{m}\left(t x_{j}+(1-t) y_{j}\right)-\ln \sum_{j=1}^{m}\left(1+t x_{j}+(1-t) y_{j}\right)$,

(ii) $g(t)=\ln \sum_{j=1}^{m}\left(t x_{j}+(1-t) y_{j}\right)^{1 / m}$,

(iii) $h(t)=\frac{1}{m} \ln \psi(t)$, where

$$
\psi(t)=\sum_{j=1}^{m} \frac{t x_{j}+(1-t) y_{j}}{1+t x_{j}+(1-t) y_{j}} .
$$

Proof. (i) Directly calculating yields

$$
f^{\prime}(t)=\sum_{j=1}^{m}\left(x_{j}-y_{j}\right)\left[\frac{1}{t x_{j}+(1-t) y_{j}}-\frac{1}{1+t x_{j}+(1-t) y_{j}}\right]
$$

and

$$
\begin{aligned}
f^{\prime \prime}(t) & =-\sum_{j=1}^{m}\left(x_{j}-y_{j}\right)^{2}\left[\frac{1}{\left(t x_{j}+(1-t) y_{j}\right)^{2}}-\frac{1}{\left(1+t x_{j}+(1-t) y_{j}\right)^{2}}\right] \\
& =-\sum_{j=1}^{m}\left(x_{j}-y_{j}\right)^{2} \frac{1+2 t x_{j}+2(1-t) y_{j}}{\left(t x_{j}+(1-t) y_{j}\right)^{2}\left(1+t x_{j}+(1-t) y_{j}\right)^{2}} .
\end{aligned}
$$

Since $f^{\prime}(t) \leq 0, f(t)$ is concave on $(0,1)$.

(ii) Directly calculating yields

$$
g^{\prime}(t)=\frac{\frac{1}{m} \sum_{j=1}^{m}\left(x_{j}-y_{j}\right)^{\frac{1}{m}-1}}{\sum_{j=1}^{m}\left(t x_{j}+(1-t) y_{j}\right)^{1 / m}}
$$

and

$$
g^{\prime \prime}(t)=-\frac{\left[\frac{1}{m} \sum_{j=1}^{m}\left(x_{j}-y_{j}\right)^{\frac{1}{m}-1}\right]^{2}}{\sum_{j=1}^{m}\left(t x_{j}+(1-t) y_{j}\right)^{2 / m}} .
$$

Since $g^{\prime \prime}(t) \leq 0, f(t)$ is concave on $(0,1)$

(iii) By computing,

$$
\begin{gathered}
h^{\prime}(t)=\frac{1}{m} \frac{\psi^{\prime}(t)}{\psi(t)} \\
h^{\prime \prime}(t)=\frac{1}{m} \frac{\psi^{\prime \prime}(t) \psi(t)-\left(\psi^{\prime}(t)\right)^{2}}{\psi^{2}(t)}
\end{gathered}
$$


where

$$
\psi^{\prime}(t)=\sum_{j=1}^{m} \frac{x_{j}-y_{j}}{\left(1+t x_{j}+(1-t) y_{j}\right)^{2}}
$$

and

$$
\psi^{\prime \prime}(t)=-\sum_{j=1}^{m} \frac{2\left(x_{j}-y_{j}\right)^{2}}{\left(1+t x_{j}+(1-t) y_{j}\right)^{3}} .
$$

Thus,

$$
\begin{aligned}
\psi^{\prime \prime}(t) \psi(t)-\left(\psi^{\prime}(t)\right)^{2} & =-\sum_{j=1}^{m} \frac{2\left(x_{j}-y_{j}\right)^{2}}{\left(1+t x_{j}+(1-t) y_{j}\right)^{3}} \sum_{j=1}^{m} \frac{t x_{j}+(1-t) y_{j}}{1+t x_{j}+(1-t) y_{j}} \\
& -\left[\sum_{j=1}^{m} \frac{x_{j}-y_{j}}{\left(1+t x_{j}+(1-t) y_{j}\right)^{2}}\right]^{2} \leq 0,
\end{aligned}
$$

and then $h^{\prime \prime}(t) \leq 0$, so $f(t)$ is concave on $(0,1)$.

The proof of Lemma 4 is completed.

Proof of Theorem A: For any $1 \leq i_{1}<\cdots<i_{k} \leq n$, by Lemma 3 and Lemma 4(i), it follows that $\ln \sum_{j=1}^{k} x_{i_{j}}-\ln \sum_{j=1}^{k}\left(1+x_{i_{j}}\right)$ is concave on $\mathbb{R}_{+}^{n}$, and then $\ln F_{n}(x, k)=\prod_{1 \leq i_{1}<\cdots<i_{k} \leq n}\left(\ln \sum_{j=1}^{k} x_{i_{j}}-\ln \sum_{j=1}^{k}\left(1+x_{i_{j}}\right)\right)$ is concave on $\mathbb{R}_{+}^{n}$.

Furthermore, it is clear that $\ln F_{n}(\boldsymbol{x}, k)$ is symmetric on $\mathbb{R}_{+}^{n}$, by Lemma 1 , it follows that $\ln F_{n}(\boldsymbol{x}, k)$ is concave on $\mathbb{R}_{+}^{n}$, and then from Lemma 2 we conclude that $F_{n}(\boldsymbol{x}, k)$ is also concave on $\mathbb{R}_{+}^{n}$.

The proof of Theorem A is completed.

Similar to the proof of Theorem A, by Lemma 4 (ii) and Lemma 4 (iii), we can prove Theorems B and C, respectively. Omitted detailed process.

\section{Acknowledgements}

Shi was supported in part by the Scientific Research Common Program of Beijing Municipal Commission of Education (KM201111417006). This article was typeset by using $\mathcal{A M S}-$ LATEX.

\section{Authors' contributions}

All authors read and approved the final manuscript.

\section{Competing interests}

The authors declare that they have no competing interests.

Received: 24 May 2011 Accepted: 17 January 2012 Published: 17 January 2012

\section{References}

1. Marshall, AW, Olkin, I: Inequalities:theory of majorization and its application. Academies Press, New York (1979)

2. Wang, B-Y: Foundations of majorization inequalities. Beijing Normal Univ. Press, Beijing, China, (Chinese) (1990)

3. Chu, Y-M, Xia, W-F, Zhao, T-H: Some properties for a class of symmetric functions and applications. J Math Inequal. 5(1):1-11 (2011)

4. Jiang, W-D: Some properties of dual form of the Hamy's symmetric function. J Math Inequal. 1(1):117-125 (2007)

5. Xia, W-F, Chu, Y-M: Schur-convexity for a class of symmetric functions and its applications. J Inequal Appl 15 (2009). vol. 2009, Article ID 493759 